\title{
FIVE LIMIT CYCLES FOR A SIMPLE CUBIC SYSTEM
}

\author{
N. G. Lloyd and J. M. Pearson
}

\begin{abstract}
We resolve the centre-focus problem for a specific class of cubic systems and determine the number of limit cycles which can bifurcate from a fine focus. We also describe the methods which we have developed to investigate these questions in general. These involve extensive use of Computer Algebra; we have chosen to use REDUCE.
\end{abstract}

\section{Introduction}

Suppose that $P$ and $Q$ are polynomials and that the origin is a critical point of focus type for the system

$$
\begin{aligned}
& \dot{x}=P(x, y), \\
& \dot{y}=Q(x, y) .
\end{aligned}
$$

We are concerned with two closely related questions, both of which are significant elements in work on Hilbert's sixteenth problem. The first is the number of limit cycles which bifurcate from a critical point and the second is the derivation of necessary and sufficient conditions for a critical point to be a centre. These questions have attracted a great deal of attention over the years and our approach involves extensive use of Computer Algebra. They are intertwined issues: in particular, an understanding of the centre conditions is required to resolve the question of bifurcation. The problem of distinguishing between a centre and a focus is of independent interest; it has been extensively studied over the years and is closely related to the question of integrability.

Research on Hilbert's sixteenth problem in general usually proceeds by the investigation of particular classes of polynomial systems. Much effort has been devoted in recent years to the investigation of various systems 
in which $P$ and $Q$ are cubic polynomials. In this talk we consider the class of systems

$$
\begin{aligned}
& \dot{x}=\lambda x+y+A x^{2}+B x y, \\
& \dot{y}=-x+\lambda y+K x^{2}+L x y+M y^{2}+N x^{3}
\end{aligned}
$$

and thereby illustrate our approach to the twin questions of bifurcation and integrability. The presence of a single cubic term in system (1.2) has a number of effects. In particular, we shall see that up to five limit cycles can bifurcate from a fine focus (as opposed to three for quadratic systems) and we shall require a wider range of techniques to resolve the centre-focus problem than is the case for quadratic systems.

\section{Centres and fine foci}

Since the origin is a critical point of focus type we write system (1.1) in canonical coordinates in the form

$$
\begin{aligned}
& \dot{x}=\lambda x+y+p(x, y), \\
& \dot{y}=-x+\lambda y+q(x, y),
\end{aligned}
$$

where $p$ and $q$ are polynomials with no linear terms. It is well known that there is a function $V$, analytic in a neighbourhood of the origin, such that $\dot{V}$, the rate of change of $V$ along orbits, is of the form $\eta_{2} r^{2}+\eta_{4} r^{4}+\cdots$, where $r^{2}=x^{2}+y^{2}$. The $\eta_{2 k}$ are polynomials in the coefficients arising in $p$ and $q$ and are the focal values. We say that the origin is a fine focus if $\lambda=0$ and a fine focus of order $k$ if $\eta_{2 \ell}=0$ for $\ell \leq k$ and $\eta_{2 k+2} \neq 0$; note that describing the critical point as a fine focus allows the possibility of its being a centre. The first task is to compute the focal values. We do so using our Computer Algebra procedure FINDETA which is described in [3] and which has been used extensively. The stability of the origin is determined by the sign of the first non-zero focal value and the origin is a centre if all the focal values vanish. Having computed the focal values they are then 'reduced' in the sense that the relations $\eta_{2}=\eta_{4}=\cdots=\eta_{2 k-2}=0$ are used in $\eta_{2 k}$. The polynomials so obtained are called the Liapunov quantities; this process also involves the use of Computer Algebra and can be computationally intensive (see [4]). By the Hilbert basis theorem there is $M$ such that $\eta_{2 k}=0$, for all $k$, if $\eta_{2 k}=0$ for $k \leq M$. Thus the origin is a centre if and only if all the focal values of $\eta_{2 k}$ with $k \leq M$ are zero. However, $M$ is not known a priori and therefore neither is the number of focal values required. Necessary conditions for the origin to be a centre are found by computing a certain number of focal values and reducing each modulo the earlier 
ones until the point is reached where the latest Liapunov quantity, with factors common to all previously computed Liapunov quantities removed, is necessarily non-zero.

Having obtained necessary conditions in this way their sufficiency is proved separately using a variety of methods. There are two classical results: the first is that the origin is a centre of (2.1) if $\lambda=0$ and the divergence $p_{x}+q_{y}$ is zero in a neighbourhood of the origin (that is, the system is Hamiltonian). The second is that we have a centre if the system is symmetric in a line through the origin - in particular if the system is unchanged by one of the transformations $(x, y, t) \mapsto(x,-y,-t)$ or $(x, y, t) \mapsto(-x, y,-t)$. Generalising the first of these results the origin is also a centre if $\lambda=0$ and there is a function $D$, continuously differentiable in a neighbourhood of the origin such that $(D p)_{x}+(D q)_{y}=0$. Such functions are called Dulac functions and are, of course, integrating factors. A systematic approach to finding these functions is developed in $[\mathbf{1}]$ and we describe in $[\mathbf{6}]$ how this search can be partly automated. Initially we search for polynomials $C_{r}$ which are invariant with respect to (2.1) and powers $\alpha_{r}$ such that the product $\Pi C_{r}^{\alpha_{r}}$ is a Dulac function. Suppose that $p$ and $q$ are of degree $n$ : we say that the polynomial $C$, or the algebraic curve $C=0$, is invariant if there is a polynomial $R$ of degree $n-1$ such that $\dot{C}=C R$. We extend this definition to include the possibility that $C$ is the exponential of a polynomial. When there is a Dulac function of this form there is a liouvillian first integral - that is, a first integral which involves polynomials, exponentials, logarithms and algebraic functions. In this case the system is sometimes said to be 'integrable'; it is of course a classical result that for systems of the form (2.1) the origin is a centre if and only if there is an analytic first integral.

The approach via invariant functions is a powerful method for proving that under given conditions the origin is in fact a centre (see [7], [8]). However, not all centre conditions can be obtained in this way, as seen in [5] and [6]. To complete the proof of the sufficiency of the centre conditions for (1.2) we exploit the fact that the system can be transformed to Liénard form and use the results of $[\mathbf{2}]$. These depend on the fact that, for any functions $p_{0}, p_{1}, q_{0}, q_{1}, q_{2}$ (not necessarily polynomials) with $p_{1}(0) \neq 0$, the system

$$
\begin{aligned}
& \dot{x}=p_{0}(x)+p_{1}(x) y, \\
& \dot{y}=q_{0}(x)+q_{1}(x) y+q_{2}(x) y^{2}
\end{aligned}
$$

can be transformed to a Liénard system. Under the transformation $(x, y, t) \mapsto\left(x,\left(p_{0}(x)+p_{1}(x) y\right) \psi(x), \tau\right)$, where $\frac{d t}{d \tau}=\psi$, the system $(2.2)$ 
becomes

$$
\begin{aligned}
& \dot{x}=y \\
& \dot{y}=-g(x)-f(x) y
\end{aligned}
$$

with

$$
f=-\left(p_{0}^{\prime}-p_{0} p_{1}^{\prime} p_{1}^{-1}+q_{1}-2 p_{0} p_{1}^{-1} q_{2}\right) \psi
$$

and

$$
g=\left(p_{0} q_{1}-p_{1} q_{0}-p_{0}^{2} q_{2} p_{1}^{-1}\right) \psi^{2}
$$

where

$$
\psi(x)=\left(p_{1}(x)\right)^{-1} \exp \left(-\int_{0}^{x} q_{2}(s)\left(p_{1}(s)\right)^{-1} d s\right) .
$$

Let $F(x)=\int_{0}^{x} f(s) d s$ and $G(x)=\int_{0}^{x} g(s) d s$. For system (2.3) two necessary and sufficient conditions for the origin to be a centre are given in [2]. The first is that there is an analytic function $\Phi$ with $\Phi(0)=0$ such that $G(x)=\Phi(F(x))$; this was the result used in [2]. The other, and the result which we use here, is the following.

Lemma 1. The origin is a centre for (2.2) if and only if there is an analytic function $z(x)$ with $z^{\prime}(0)=-1$ such that

$$
F(z(x))=F(x) \text { and } G(z(x))=G(x) .
$$

\section{Centre conditions}

We consider (1.2) with $N \neq 0$. When $N=0$ the differential system is quadratic and the relevant results are well known. Using FINDETA we calculate the focal values up to $\eta_{12}$. We obtain the Liapunov quantity $L(i)$ from the focal value $\eta_{2(i+1)}$ modulo $\eta_{2 j}$, where $j=1, \ldots, i$, with positive multiplicative factors removed. We note that for the origin to be a fine focus we must have $L(0)=\lambda=0$. We find $L(1)=-A B+2 A K+K L+L M$. For a fine focus of order greater than one we require $L(1)=0$. Assume for the time being that $L \neq 0$ and let $M=L^{-1}(A B-2 A K-K L)$. Then

$$
L(2)=L^{-1}(2 A+L)\left(L^{2} D_{1} N-A(3 A-L) F_{1}\right),
$$

where $D_{1}=4 A B-8 A K+2 B L+K L$ and

$$
\begin{aligned}
F_{1}=A B^{3}-6 A B^{2} K+12 A B K^{2} & -A B L^{2}-8 A K^{3}+2 A K L^{2}-B^{3} L \\
& +3 B^{2} K L+B L^{3}-4 K^{3} L-K L^{3}
\end{aligned}
$$


When $M$ is given as above, $(2 A+L)$ is a factor of $\eta_{8}, \eta_{10}, \eta_{12}$, suggesting that $L=-2 A, B=-2 M$ is a condition for the origin to be a centre. In fact when this condition holds the divergence of the vector field is zero and the origin is a centre.

Assume that $L(2 A+L) D_{1} \neq 0$ and let $N=L^{-2} D_{1}^{-1} A(3 A-L) F_{1}$. Then $L(2)=0$ and the origin is a fine focus of order greater than two or it is a centre. We have

$$
L(3)=-\left(L D_{1}\right)^{-1}(2 A+L) A C_{1} F_{1}\left(E_{2} K^{2}+E_{1} K+E_{0}\right),
$$

where

$$
\begin{aligned}
& C_{1}=A B-2 A K-2 B L-K L, \\
& E_{0}=B^{2}(3 A-L)\left(11 A^{2}+14 A L-L^{2}\right), \\
& E_{1}=-2 B(2 A+L)\left(33 A^{2}-2 A L-L^{2}\right), \\
& E_{2}=2 A\left(66 A^{2}-4 A L-L^{2}\right) .
\end{aligned}
$$

Now if $A F_{1}=0$ then $N=0$, which is excluded. We find $C_{1}$ is a factor of $\eta_{10}, \eta_{12}$ with $M, N$ as given above, which suggests that

$$
\left\{\begin{array}{l}
M=2 B,(2 A+L) K-B(A-2 L)=0 \\
25(2 A+L) N=(L-3 A)\left(8 A^{2}-2 A L-25 B^{2}-3 L^{2}\right)
\end{array}\right.
$$

is another condition for the origin to be a centre.

In the following assume that $L(2 A+L) C_{1} D_{1} \neq 0$. For the origin to be a fine focus of order at least four we must have $E_{2} K^{2}+E_{1} K+E_{0}=0$. Assume that $E_{2} \neq 0$, if $E_{2}=0$ there are no conditions for the origin to be a centre that are not covered elsewhere in this section. Let $K=\frac{1}{2}\left(-E_{1}+2 B L R_{0}\right) E_{2}^{-1}$, then $L(4)=\left(66 A^{2}-4 A L-L^{2}\right)^{-1}(2 A+L)(3 A-L) C_{1} F_{1} B\left(S R_{0}+T\right)$, where $S, T$ are homogeneous polynomials in $A, L$ of degrees 11,13 respectively with integer coefficients of magnitude up to $10^{11}$ and

$$
R_{0}^{2}=2871 A^{4}-570 A^{3} L-68 A^{2} L^{2}+10 A L^{3}+L^{4} .
$$

Under current assumptions the origin can be a fine focus of order five only if $S R_{0}+T=0$. Assume that $S \neq 0$ (if $S=T=0$ then $A=L=0$ which violates current hypotheses) and let $R_{0}=-T S^{-1}$. Now for $R_{0}$ to satisfy (3.2) we require

$$
\Gamma=(9 A+L)(5 A-L)(7 A+L)(15 A+L) \phi=0,
$$


where $\phi=4506 A^{4}-1140 A^{3} L+149 A^{2} L^{2}+2 A L^{3}-L^{4}$. When $\phi \neq 0$ but $\Gamma=0$ we find $\eta_{12}=0$. This suggests that the origin is a centre if

$$
\begin{aligned}
& L=-9 A, \quad K=-4 B, \quad M=3 B, \quad N=-4\left(3 A^{2}+B^{2}\right) \\
& \text { or if } L=5 A, \quad K=-2 B, \quad M=3 B, \quad N=-\left(A^{2}+B^{2}\right) \\
& \text { or if } L=-7 A, \quad K=4 B, \quad M=-3 B, \quad N=-4\left(A^{2}+B^{2}\right) \\
& \text { or if } L=-15 A, \quad K=-2 B, \quad 3 M=5 B, \quad N=-45 A^{2}-B^{2} \text {. }
\end{aligned}
$$

When none of these conditions hold and $\phi=0$ we have

$$
\begin{gathered}
L(5)=-\left(A L S D_{1}\right)^{-1}\left(66 A^{2}-4 A L-L^{2}\right)^{-1}(2 A+L)(5 A-L)(7 A+L) \\
(15 A+L)\left(151 A^{2}-14 A L-L^{2}\right) C_{1} F_{1}\left(N_{1} B^{2}+N_{2}\right)
\end{gathered}
$$

where $N_{1}, N_{2}$ are bivariate, homogeneous polynomials of degrees 11,13 respectively. Under current assumptions $L(5)$ is non-zero.

In order to determine all necessary conditions for which the origin is a centre for (1.2) we return to those cases that were excluded in the preceding argument. When $L=0$, we find that $A$ is a factor of all the calculated focal values. This suggests that $A=L=0$ is a condition for the origin to be a centre. Assume that $A \neq 0$; then $L(1)=0$ if $B=2 K$ and we have $L(2)=A\left(3 M_{1}+M_{2} N\right)$, where $M_{1}=A^{2} M+2 K^{3}+3 K^{2} M-M^{3}, M_{2}=9 K+4 M$. For the time being assume that $M_{2} \neq 0$; now $L(2)=0$ if $N=-3 M_{1} M_{2}^{-1}$ and then $L(3)=A(4 K-M) M_{1}\left(15 K^{2}+44 K M+11 M^{2}\right)$. With $L=0$, $M=4 K, B=2 K, 25 N=-6\left(2 A^{2}-25 K^{2}\right)$ the relationships of (3.1) are satisfied. If $M_{1}=0$ then $N=0$, which is excluded as is $A=0$. If $A(4 K-M) M_{1} \neq 0$ then $L(3)=L(4)=0$ if and only if $K=M=0$, which also violates current assumptions. On the other hand if $M_{2}=0$ then $L(2)=A K\left(425 K^{2}-144 A^{2}\right)$ and $K$ is a factor of $\eta_{8}, \eta_{10}, \eta_{12}$ suggesting that the origin is a centre if $B=K=L=M=0$. There are no other new conditions arising in the case $L=0$.

Consider $D_{1}=4 A B-8 A K+2 B L+K L=0$. Assume that $2 A+L \neq 0$ and let $B=\frac{1}{2} K(8 A-L)(2 A+L)^{-1}$. The case $L=0$ is covered above so we can assume that $L \neq 0$ and let $M=L^{-1}(A B-2 A K-K L)$ such that $L(1)=0$. Under current assumptions $L(2)$ is zero if $A K(3 A-L) G_{1}=0$, where $G_{1}$ is a polynomial of degree three in $A, K$ and $L$, and $A K$ is a factor of $\eta_{8}, \eta_{10}, \eta_{12}$. This suggests that $A=0, K=-M=-2 B$ (which is covered by (3.1)) and $B=K=M=0$ are conditions for the origin to be a centre. When $L=3 A$ or $G_{1}=0$ no further conditions are found. Similarly, when $L=-2 A$ no new centre conditions are obtained.

We summarize the necessary conditions for the origin to be a centre in the following result. 
Theorem 2. The origin is a centre for (3.1), with $\lambda=0, N \neq 0$, if and only if one of the following conditions holds:

(i) $B=-2 M, L=-2 A$;

(ii) $A=L=0$;

(iii) $B=K=M=0$;

(iv) $M=2 B,(2 A+L) K-(A-2 L) B=0$, $25(2 A+L) N+(3 A-L)\left(8 A^{2}-2 A L-25 B^{2}-3 L^{2}\right)=0 ;$

(v) $K=-4 B, L=-9 A, M=3 B, N=-4\left(3 A^{2}+B^{2}\right)$;

(vi) $K=-2 B, L=5 A, M=3 B, N=-\left(A^{2}+B^{2}\right)$;

(vii) $K=4 B, L=-7 A, M=-3 B, N=-4\left(A^{2}+B^{2}\right)$;

(viii) $K=-2 B, L=-15 A, 3 M=5 B, N=-45 A^{2}-B^{2}$.

Proof: The necessity of conditions (i)-(viii) has been shown in the argument leading to Theorem 2. We have already stated that when condition (i) of Theorem 2 holds, the divergence of the vector field is zero. When conditions (ii) or (iii) hold the vector field is symmetric, in the $x$ - or $y$-axis respectively, and thus the origin is a centre. The sufficiency of conditions (iv)-(vii) is proved by finding invariant curves and constructing appropriate Dulac functions as outlined in section 2 and described in detail in [6]. Using the result of part (ii) of Lemma 2.2 in [6], regarding factors of the highest order terms of an invariant curve, we know that any invariant polynomial of degree $n$ of (1.2) will be of the form $x^{n}+$ lower order terms. In each case only one invariant curve is required.

Specifically, when (iv) holds, $C_{1}^{\alpha}$ is a Dulac function where

$$
C_{1}=\left(-8 A^{2}+2 A L+25 B^{2}+3 L^{2}\right) x^{2}+50 B x-10(2 A+L) y+25
$$

and $\alpha=-5 / 2$. For conditions (v), (vi) or (vii) an invariant degree three curve is appropriate. Again, writing the Dulac function as $C_{1}^{\alpha}$, we have

$C_{1}=8 B\left(3 A^{2}+B^{2}\right) x^{3}+3\left(3 A^{2}+4 B^{2}\right) x^{2}+18 A B x y-3 B^{2} y^{2}+6 B x+6 A y+1$,

$\alpha=-7 / 6$ when condition $(\mathrm{v})$ holds,

$$
C_{1}=B\left(A^{2}+B^{2}\right) x^{3}+3\left(A^{2}+B^{2}\right) x^{2}+3 B x-3 A y+1,
$$

$\alpha=-7 / 3$ when condition (vi) holds and

$C_{1}=-8 B\left(A^{2}+B^{2}\right) x^{3}+3\left(A^{2}+4 B^{2}\right) x^{2}-18 A B x y-9 B^{2} y^{2}-6 B x+6 A y+1$, 
$\alpha=-5 / 6$ when condition (vii) holds.

Extensive searching failed to yield an appropriate invariant curve for condition (viii) without imposing additional relationships between the coefficients. Here transformation to Liénard form proves more judicious.

To prove the sufficiency of condition (viii) we use Lemma 1 . The corresponding Liénard system $(2.3)$ has $\psi(x)=(1+B x)^{-8 / 3}$,

$$
f(x)=13 A x(1+B x)^{-11 / 3}\left(1+\frac{4}{3} B x\right)
$$

and

$$
g(x)=x(1+B x)^{-19 / 3}\left[(1+B x)^{4}+\frac{5}{3} A^{2} x^{2}\left(27 B^{2} x^{2}+44 B x+18\right)\right] .
$$

We use REDUCE to obtain $F$ and $G$, the integrals of $f$ and $g$, respectively. Let $1+B x=u^{3}$ and $1+B y=v^{3}$. It is found that

$$
\Omega=4 u^{3} v^{3}-u^{3}-v^{3}-u^{2} v-u v^{2}
$$

is a common factor of $F(u)-F(v)$ and $G(u)-G(v)$. In terms of $x$ and $y$,

$\Omega=4 B^{2} x y+3 B(x+y)+2-(B x+1)^{2 / 3}(B y+1)^{1 / 3}-(B x+1)^{1 / 3}(B y+1)^{2 / 3}$.

Now, when $x=y=0, \Omega_{x}=\Omega_{y}$. By the Implicit Function Theorem there is $y(x)$ with $y^{\prime}(x)=-1$ such that $F(x)=F(y(x))$ and $G(x)=G(y(x))$. The origin is therefore a centre by Lemma 1 .

\section{Limit Cycles}

In section 3 we have given the conditions under which the origin is a centre for (1.2). In arriving at our conclusions we found that the highest possible order for a fine focus at the origin is five.

Theorem 3. The origin is a fine focus of maximum order five for (1.2). It is of order five if

$$
\begin{gathered}
\lambda=0, M=L^{-1}(A B-2 A K-K L), N=L^{-2} D_{1}^{-1} A(3 A-L) F_{1}, \\
K=\frac{1}{2} E_{2}^{-1}\left(-E_{1}+2 B L R_{0}\right) \\
4506 A^{4}-1140 A^{3} L+149 A^{2} L^{2}+2 A L^{3}-L^{4}=0 \\
R_{0}=-6 A^{2}\left(38657056203 A^{3}-7050603615 A^{2} L-75427866 A L^{2}\right. \\
\left.+39349768 L^{3}\right) /\left(2961753180 A^{3}-121564362 A^{2} L\right. \\
\left.-19237927 A L^{2}+177687 L^{3}\right)
\end{gathered}
$$


and $N L D_{1} E_{2} S \neq 0$.

We proceed to show that five limit cycles can be bifurcated in this instance. First we summarise the Liapunov quantities obtained

$$
\begin{aligned}
L(0)= & \lambda, \\
L(1)= & -A B+2 A K+K L+L M, \\
L(2)= & (2 A+L)\left(L^{2} D_{1} N-A(3 A-L) F_{1}\right) / L, \\
L(3)= & -(2 A+L) A C_{1} F_{1}\left(E_{2} K^{2}+E_{1} K+E_{0}\right) / L D_{1}, \\
L(4)= & (2 A+L)(3 A-L) C_{1} F_{1} B\left(S R_{0}+T\right) / H_{1}, \\
L(5)= & -(2 A+L)(5 A-L)(7 A+L)(15 A+L) H_{2} C_{1} F_{1}\left(N_{1} B^{2}+N_{2}\right) \\
& / A L S D_{1} H_{1},
\end{aligned}
$$

where $H_{1}=66 A^{2}-4 A L-L^{2}, H_{2}=151 A^{2}-14 A L-L^{2}$. We consider $\phi$ as a polynomial in $A / L$; it has two real roots. In particular there is a root, $u^{*}$ say, in the interval $I=(-0.06780395,-0.06780393)$. In $I$, $C_{1} F_{1}>0,66 A^{2}-4 A L-L^{2}<0,151 A^{2}-14 A L-L^{2}>0, S>0$, $T<0$ and $D_{1}<0$. Therefore at $u^{*}, L(5)$ is positive, so if we perturb $A / L$ such that $L(4)$ becomes negative, at the same time ensuring that $L(0), L(1), L(2), L(3)$ remain zero, a limit cycle bifurcates. For this we require $B\left(S R_{0}+T\right)<0$. At $u^{*}, R_{0}>0$. In addition $R_{0}$ is increasing in $I$. If $B<0$ then we increase $A / L$, conversely if $B>0$ we decrease $A / L$, at the same time adjusting $K, N$ and $M$ appropriately. For the second limit cycle $K$ is decreased such that $L(3)$ becomes positive, that is $-(2 A+L)\left(E_{2} K^{2}+E_{1} K+E_{0}\right)>0$, at the same time adjusting $N, M$ so that $L(1)=L(2)=0$. If we now increase $N$ then $L(2)<0$ and a third limit cycle bifurcates. Next we perturb $M$ such that $L(1)=-A B+2 A K+K L+L M>0$; if $L>0$ we increase $M$, if $L<0$ we decrease $M$. A fourth limit cycle bifurcates. The fifth and final limit cycle is obtained by introducing a negative $\lambda$.

This example demonstrates the value of Computer Algebra in resolving the centre-focus problem and in the investigation of limit cycles which bifurcate from a fine focus. It also shows that the addition of only one cubic term has a significant effect on both these issues.

Acknowledgements. We are grateful to Professor A. P. Sadovskii for drawing our attention to system (1.2). We also wish to express our thanks to Professor John Fitch and the University of Bath for allowing us to use their computing facilities. 


\title{
References
}

1. C. J. Christopher, Invariant algebraic curves and conditions for a centre, Proc. Roy. Soc. Edinburgh 124A (1994), 1209-1229.

2. C. J. Christopher, N. G. Lloyd and J. M. Pearson, On Cherkas's method for centre conditions, Nonlinear World 2 (1995), 459-469.

3. N. G. Lloyd and J. M. Pearson, REDUCE and the bifurcation of limit cycles, J. Symbolic Comput. 9 (1990), 215-224.

4. N. G. Lloyd AND J. M. Pearson, Computing centre conditions for certain cubic systems, J. Comp. Appl. Math. 40 (1992), 323-336.

5. N. G. Lloyd and J. M. Pearson, Limit cycles and centres: an example, in "Differential Equations, Dynamical Systems and Control Science," K. D. Elworthy, W. N. Everitt and E. B. Lee, eds., Marcel Dekker, New York, 1993, pp. 143-158.

6. J. M. Pearson, N. G. Lloyd and C. J. Christopher, Algorithmic derivation of centre conditions, SIAM Rev. 38 (1996), 619-636.

7. D. Schlomiuk, Elementary first integrals and algebraic invariant curves of differential equations, Exposition. Math. 11 (1993), 433-454.

8. D. Schlomiuk, Algebraic particular integrals, integrability and the problem of the center, Trans. Amer. Math. Soc. 338 (1993), 799-841.

\author{
Department of Mathematics \\ University of Wales \\ Aberystwyth \\ Ceredigion SY23 3BZ \\ U.K. \\ e-mail: ngl@aber.ac.uk \\ e-mail: jmp@aber.ac.uk
}

Primera versió rebuda el 30 de Novembre de 1996, darrera versió rebuda el 17 de Febrer de 1997 